\title{
Research on Design and Application Based on Constant Temperature Performance in Clothing Structure
}

\author{
Yang Qingmei ${ }^{1}$, Wang Lihui ${ }^{1}$ and Zhang Yansong ${ }^{2}$ \\ 1,2Dalian Polytechnic University School of Fashion and Design ,Liaoning Dalian,China
}

\begin{abstract}
With the development of the times, people's living standards have gradually improved, and the requirements for clothing have also increased. This is a thermostatic clothing suitable for somatosensory and microclimate environments in high temperature and hot and low temperature and cold environments. It uses functional materials to strengthen the temperature regulation of the thermostatic energy storage layer and extend the effective working time of the thermostatic clothing. This paper conducts in-depth research on the clothing structure, combines the design and application of constant temperature performance and discovers the relationship between the two, promotes the effective integration of the two, improves the aesthetic and practical value of clothing, and promotes the simultaneous development of my country's clothing industry.
\end{abstract}

\section{Introduction}

The clothing industry is based on the clothing structure to determine a reasonable production process, which fully demonstrates the artistry and aesthetics of clothing. Since the 21 st century, the vigorous economic development has brought about radical changes in social living standards, and people's yearning for the quality of life and consumption patterns have also evolved with the times. On the one hand, the formation of a diversified and subdivided structure model of clothing has made more consumers gradually concerned about the feasibility of clothing design and innovative fashion; On the other hand, with the advancement of science and technology, new fabrics continue to appear, clothing its functional design has become closer to modern life and has become a new consumer hot spot for the public [1]. The functional design of clothing reflects people's most basic demands on the way of dressing. It is not only the source of clothing development, but also the trend of clothing.

\section{Development status at home and abroad}

In 2002, Li Jun of Donghua University proposed a new method for predicting the comfort of dressing temperature, which can determine the physiological changes and subjective psychological feelings of different parts of the human body when they are subjected to the same degree of cold stimulation. The differences were studied. And using multiple comparisons to analyze the response law of main body parts to skin temperature changes caused by cold stimulation, it is concluded that after each part of the body is subjected to the same degree of cold stimulation, there is a significant difference between the maximum changes in skin temperature. The difference is decreasing in order of left chest and left abdomen, left thigh and right calf, left side of back waist, front of right upper arm and left forearm, left side of back, and front of right thigh Based on the analysis of subjective feelings, the sensitivity ranks are: waist, back, chest, thighs, upper limbs, and calves. All this shows that a reasonable dressing method needs to consider effective coverage of sensitive parts and parts with the largest skin temperature changes [2].

In his research on the relationship between the warmth retention of clothing materials and the thermal resistance of clothing, Li Dongping of Shanghai Textile School concluded that the thermal resistance of clothing is different from the thermal resistance of fabrics, and there is no significant correlation between the two, and pointed out that multiple pieces of clothing The thermal resistance value of the pull-on is always less than the sum of the thermal resistance of each single piece. The more the number of pull-on pieces, the smaller the increment of the thermal resistance value of the garment after pull-on. At the same time, he also pointed out that pull-on garments should have a reasonable wearing sequence, and that the inner tightness and outer looseness are beneficial to improve the warmth of the garment [3].In 2004, Joo-Young Lee compared the metabolic rate and subjective thermal response of the human body under two dressing styles (S-type and L-type) in a neutral and cold environment [4]. The results show that the human body wearing the S-type consumes more energy than the body wearing the L-type, but there is no difference in subjective thermal response.

The research goal of this article is to explore the human body constant temperature adjustment mode of human body clothing and the difference of the physiological and subjective feelings of different 
dressing methods, so as to obtain the reasonable design of the best constant temperature performance in the clothing structure.

\section{The transfer mechanism between human body, clothing and environment}

The basic condition for human survival is to maintain a body temperature of around 37 degrees. If the temperature is exceeded or below this temperature for a long time, it will harm health and even endanger life. Body temperature depends on the balance between the body's heat production and heat dissipation. Normal body temperature is relatively constant, that is, under the control of the body temperature regulation mechanism, the two processes of heat production and heat dissipation are dynamically balanced.

Because clothing has the dual functions of assisting the human body to carry out thermal regulation and protecting the body from direct damage from the external environment, the human body can well control the heat exchange between the skin and the external environment in a thermal environment. In view of this heavy effect, the integrated system of "human body-clothing-environment" came into being. The human body mainly uses conduction, convection, and radiation to dissipate heat to the surrounding environment and the evaporation of sweat on the human skin surface to dissipate heat. Clothing is used as a buffer between the environment and the human body, especially in a cold environment. Clothing can well control the heat exchange between the environment and the skin, and adjust the thermal resistance and moisture resistance during the heat exchange between the human body and the environment. The role of clothing is indispensable.

\section{Application design of constant temperature performance in clothing design structure}

The combination of various parts of the clothing is called the clothing structure. The structure has a certain cumbersomeness and various forms. According to the material of the clothing, various elements can be formed. The clothing structure involves surface materials, fillers, etc. The above several links are very important in the production process, so various links are involved in the garment structure to help the garment structure show its integrity. At the same time, pay attention to the characteristics and design sense expressed by the designer in the design process .

\subsection{Introduction of constant temperature materials in clothing design}

Constant control performance adopts special fiber material, mainly spliced design, processing and application in key parts of the garment structure such as joints, with flexibility and convenience. Heating fiber is a brand-new material that heats up on its own and warms the body, and its water absorption is far superior to other types of fiber. Traditional heat preservation is mainly to prevent the escape of heat emitted by the body. At present, the so-called self-heating fiber has limited functions in heating, because starting from the law of conservation of matter, the heat energy that appears can only be obtained from the human skin breathing. At present, the fever of thermal underwear is about three to five degrees. In China's textile industry, there is no defined standard for heating fibers. Refrigeration superabsorbent fiber is mainly aimed at the refrigeration clothing market. Its main advantage is to reduce the heat response and fatigue of the wearer, and to give better comfort and performance acceptance, and it can be washed multiple times. At present, the fiber has been made into various clothing, which can be worn close to the body, so that the temperature of the wearer will drop by about six degrees when exposed to thermal stress. The fire department is used as the lining of the fire protection suit. Quick-drying fiber, constant temperature and warmth, can help the body quickly excrete sweat during activities, prevent sweat from staying on the skin surface to dry naturally, take away body heat, so that the body maintains a certain constant temperature.

\subsection{Application of constant temperature performance in clothing structure}

Since the 21 st century, with the rapid development of new materials and new processes and the development of high-performance fiber raw materials, the classification of functional clothing has gradually become clear. The new functional fabric integrates high-tech fiber and high-tech technology to meet the needs of the human body in a special environment. At present, the functional characteristics of the fiber can be achieved through molecular design and structural design. Among them, the fabric composite lamination technology successfully achieved the superposition and efficiency enhancement of functions without destroying the respective functions. The development of computer technology and information processing technology has provided a broad space for the development of functional clothing with multi-functionality and compounding. Intelligent and active functional clothing has also been launched on the market.

The clothing series with constant control performance is suitable for use in hot summer and severe cold areas in winter. The dark areas are made of fiber materials with constant temperature performance. The waist, sides, shaft joints, shoulders, and necklines of the key parts of the human body are selected from constant temperature materials. Application design meets the user's psychological needs of temperature and demeanor (the structure pattern is shown in Figure 1-2). 


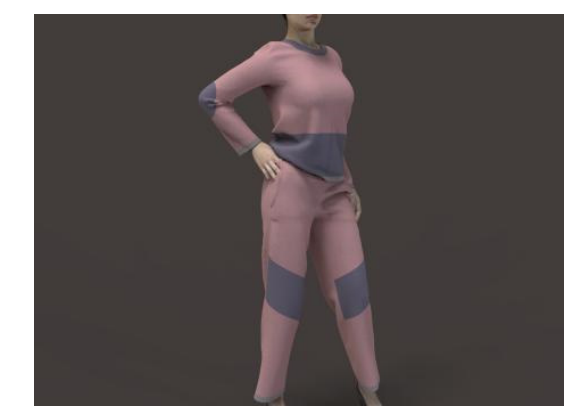

Figure1. Front thermostatic structure rendering

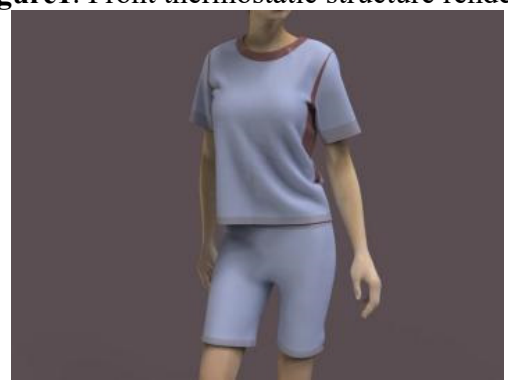

Figure 2. Front constant-drop rendering

\subsection{Characteristic analysis of constant temperature performance in clothing structure}

The needs of social life are diversified, and more people are beginning to pay attention to their own health, their own health care, and more of their own needs. The physical, basic material needs, and the inner psychological needs are generated after reaching the living conditions. Demand should not be underestimated.

Constant control performance. New clothing textile technology is used in key parts of the clothing structure. The cooling super absorbent fiber, heating fiber, and quick-drying fiber are applied to the clothing structure. It has cooling, temperature increase, temperature control, antibacterial deodorization, mildew resistance, anti-mite, Various characteristics such as moisture absorption, breathability, softness and comfort, low carbon and environmental protection. Excellent performance, soft and comfortable, strong moisture absorption and breathability. It also possesses health care, anti-ultraviolet, anti-static properties. The hemp non-twisted composite material that combines the advantages of the two has more advantages in comprehensive performance.

\section{Development prospects}

In the 21 st century with the rapid development of science and technology, it is hoped that functional design can spread across all corners of people's lives, so that functional design products can benefit people's livelihood based on the development of the times, better reduce the burden of life, improve the quality of life, and enable consumers to live in life. Realizing the power of science and technology, let the sense of design innovation take root in people's hearts, so that the design will develop more benignly, and it will also play a role in promoting the development of the country's economic strength.

\section{Conclusion}

The research and development of temperature control clothing in the market is still in its infancy, and the pressure and competition in the industry are small; the exploration of constant control performance in the clothing field is ahead of the times, and it is easy to achieve first-mover advantage. In this paper, the design of the constant temperature function improves the structure of the clothing, using existing functional clothing and functional fabrics, the emergence of functional clothing meets more market needs, from thermal underwear to body-sculpting clothing, as well as high the technological content of nano-clothing, etc., greatly meets the needs of consumers for various forms of constant-temperature clothing at all levels, and can provide a certain reference basis for the functional design of future clothing.

\section{References}

1. Li Chenglu, L. (2015) On the functional design innovation of home clothing,.D. Xi'an Polytechnic University, Master.

2. Li Jun, L. (2002) Research on the difference of skin cold sensation between parts of the human body-multiple comparisons of local skin temperature changes, Donghua University J.,(Natural Science Edition).

3. Li Dongping,L. (1998) The relationship between the warmth retention of clothing materials and the thermal resistance of clothing. J. Journal of Textile Research., 5:292-294.

4. Joo-Young Lee, J,. (2004) Jeong-Wha Choi. Influence of clothing types on metabolic, thermal and subjective responses in a cool environment. Jounal of Thermal Biology., 29:221-229. 